\title{
MD simulation of phase transformations due to nanoscale cutting on silicon monocrystals with diamond tip
}

\author{
Qiheng Tang and Fenghua Chen \\ State Key Laboratory of Nonlinear Mechanics, Chinese Academy of Sciences, Beijing \\ 100080, People's Republic of China \\ E-mail: qhtang@1nm.imech.ac.cn
}

Received 25 April 2006, in final form 28 June 2006

Published 4 August 2006

Online at stacks.iop.org/JPhysD/39/3674

\begin{abstract}
A three-dimensional molecular dynamics simulation is performed to study atomic force microscopy cutting on silicon monocrystal surface. The displacive phase transformation from the four-coordinated diamond cubic phase to the six-coordinated $\beta$-silicon phase is observed due to localized high pressure. During phase transformation, atoms are of high potential and temperature, and the values of pressure and temperature are in good agreement with those according to the phase diagram.
\end{abstract}

(Some figures in this article are in colour only in the electronic version)

\section{Introduction}

Interest is rising in processing directly the material surface by means of an atomic force microscope (AFM)-based lithographic technique [1-3], with the significant advantage of fabrication resolution extending to a nanometre regime. However, an open question is still the nature of the deformation mechanism of the atomic scale. Molecular dynamics (MD) simulations may trace the dynamic behaviour of atoms and, therefore, shed light on the understanding of the underlying deformation mechanism at the atomic scale.

Some experimental studies revealed the phase transformation of silicon from a diamond- to a $\beta$ - structure [4-7]. The metallic properties of $\beta$-silicon have been inferred by the fall in the resistivity of silicon by a few orders of magnitude during indentation. By applying ex situ transmission electron microscopy (TEM) and micro-Raman spectroscopy to examine the behaviour of silicon, Mann et al [6] observed the contact resistance to be low because of the presence of the metallic $\beta$ - phase silicon during nanoindentation loading, whereas the semimetallic phase and the rhombohedral phase are formed during unloading. Lorenz [7] and Kailer et al [8] studied phase transformation of silicon with TEM and showed that metallic $\beta$ - silicon is produced near the interface between the diamond indenter and silicon. The experimental results have provided us with a wealth of information and credible basis for further studies in phase transformation. Unfortunately, the experimental picture cannot display the atomic process involved in phase transformation. The MD approach has been applied to study various dynamic deformation processes on the atomic scale, e.g. indentation, adhesion, friction, surface defect and cutting $[9,10]$.

Cheong and Zhang [11] studied the phase transformation in silicon monocrystals via MD. Two kinds of structural transition exist, i.e. from diamond to a tetragonal-body-centre structure upon the loading of the indenter, and then from a tetragonal-body-centre to an amorphous structure upon unloading. However, the physical and mechanical behaviours during the phase transformation are unknown.

In this paper, a MD simulation under the canonical ensemble (NVT) is applied to study the phase transformation of monocrystal silicon during the cutting process. The motivation for the work is to understand the phase transition in silicon during cutting process and to give a comparison with that of indentation also by MD simulation. Special attention is paid to the deformation, temperature, pressure and energy variation of phase transformation. The plan of the paper is as follows: in the next section the details of the simulation method will be described; in section 3 the simulation results and discussion are presented and finally the conclusion will be drawn in section 4. 


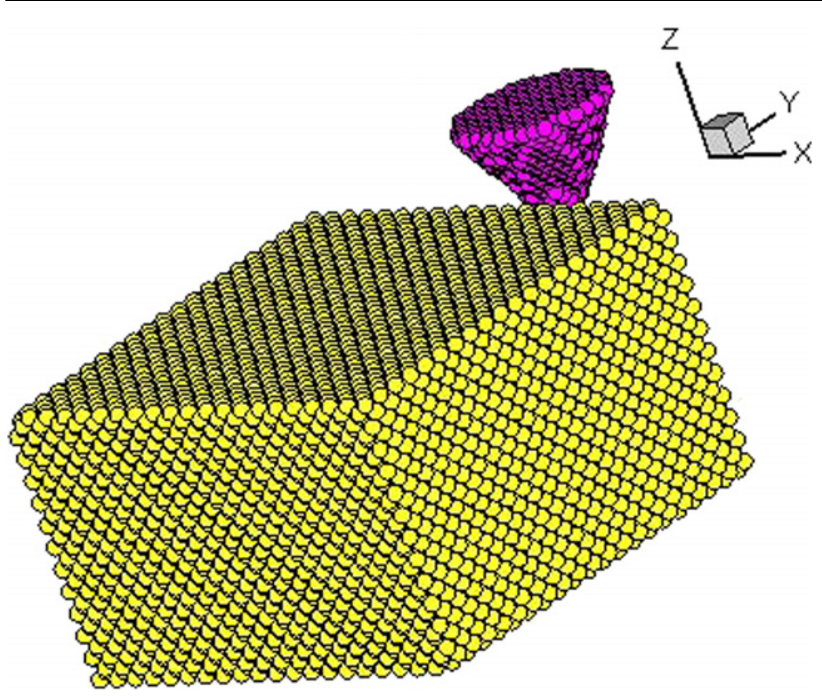

Figure 1. MD simulation model of a silicon monocrystal and a diamond pin tool. The size of workpiece silicon is $20 \times 20 \times 12 \mathrm{~nm}^{3}$. The silicon crystal orientation is $\mathrm{x}$ [100], $\mathrm{y}[010]$, $\mathrm{z}[001]$. The cutting velocity of pin tool is $110 \mathrm{~m} \mathrm{~s}^{-1}$.

\section{Computation and modelling}

\subsection{Initial model}

In the current simulation, a workpiece and a pin tool are assumed to consist of a monocrystalline silicon and a rigid diamond, respectively, as shown in figure 1 . The dimensions of the silicon workpiece are constructed sufficiently large to eliminate boundary effects. According to the simulations of Fang et al [12], the size of a workpiece which is $20 \times$ $20 \times 12 \times \mathrm{nm}^{3}$ along $x, y$ and $z$ directions is enough. The diamond tool has a $60^{\circ}$ tool angle with a tip radius of $3.4 \AA$. The workpiece is made up of 38400 silicon atoms and the tool is made of 1945 carbon atoms. The periodic boundary condition [13] is used in the transverse $x$ direction and one layer of atoms on the left and three layers of atoms on the bottom sides of silicon are fixed in space. The pin tool is moved on the silicon surface (001) along the negative $y$ direction [0ㅣㅣㄹ.

\subsection{Interatomic potentials}

There are several categories of the existing potential models for silicon, e.g. the Tersoff type [14,15] and the Stillinger-Weber two- and three-body potentials [16]. The Tersoff potential has been widely used in MD simulation.

For covalent silicon, bond strength and the directionality of bond are important. The Tersoff potential can well describe the properties of silicon-silicon and carbon-carbon and give reliable results of simulation. Hence, the Tersoff potential is selected in our work to indicate the interaction between silicon atoms $[14,15]$.

The interaction between the silicon atoms and the diamond pin tool atoms is modelled by the Morse potential [11] given by

$\phi\left(r_{i j}\right)=\lambda_{1} D\left[\exp \left(-2 \lambda_{2} \alpha\left(r_{i j}-r_{0}\right)\right)-2 \exp \left(-\lambda_{2} \alpha\left(r_{i j}-r_{0}\right)\right)\right]$,

where parameters such as $D, \alpha$ and $r_{0}$ are shown in table 1 .
Table 1. Parameters in Morse potential.

\begin{tabular}{ll}
\hline Parameters & $\mathrm{C}-\mathrm{Si}$ \\
\hline$D(\mathrm{eV})$ & 0.435 \\
$\alpha\left(\mathrm{nm}^{-1}\right)$ & 46.487 \\
$r_{0}$ & 0.19475 \\
$\lambda_{1}$ & 1 \\
$\lambda_{2}$ & 1 \\
\hline
\end{tabular}

\subsection{Temperature condition and loading method}

Initial velocities of atoms are specified based on the Maxwellian distribution corresponding to a given temperature, $T_{0}=30 \mathrm{~K}$, and the magnitudes may be adjusted so as to keep constant temperature in the system according to

$v_{i}^{\text {new }}=\left\{\frac{3 N \kappa_{\mathrm{B}} T_{0}}{2}\left[\sum_{i=1}^{N} \frac{m_{i}\left(v_{i}^{\text {old }}\right)^{2}}{2}\right]^{-1}\right\}_{i}^{1 / 2} v_{i}^{\text {old }}$,

where $v_{i}$ is the velocity of atom $i, T_{0}$ is a specified temperature, $\kappa_{\mathrm{B}}$ is Boltzmann's constant and $N$ is the number of atoms of workpiece in which fixed atoms are excluded [12]. The time integration of motion is performed by the fifth Gear's predictor-corrector method [17], time step $\Delta t=1.0 \mathrm{fs}$.

\section{Results and discussion}

The simulation system consists of a silicon workpiece and a diamond tool. The experiment shows that the silicon hardness is of $9.3 \mathrm{GPa}$ [18] and of diamond is $78.96 \mathrm{GPa}$ [19]; one can assume that the diamond tip does not deform in the process of cutting the workpiece surface. After $4000 \mathrm{MD}$ steps relaxation with the environment temperature $30 \mathrm{~K}$, a stable structure of workpiece is obtained. And the pin tool begins to cut along the $-Y$ direction [010] with velocity $110 \mathrm{~m} \mathrm{~s}^{-1}$. It is noted that the cutting speed is unrealistically high in comparison with AFM experiments. However, the previous results [13, 20,21] confirmed a little difference in the deformation characteristics such as the surface quality between 20 and $200 \mathrm{~m} \mathrm{~s}^{-1}$ cutting speed. The depth of the diamond tip plunging into the silicon workpiece is $2 \mathrm{~nm}$ which is similar to that used by Fang et al [12]. Some simulation results and analysis are shown as follows.

\subsection{Analysis of phase transition of the atomic scale}

The progress of the tool at $20 \mathrm{ps}$ intervals is shown in figures $2(a)-(d)$. The pile-up of scribing material on two sides is observed and a wedge-shaped chip is formed at 20000 steps or $20 \mathrm{ps}$. The side flow is also formed on the two sides of the pin tool and the height of the side flow can be measured. Dripping of the chip atoms from the workpiece is seen in figure $2(d)$.

Figure 3 shows a typical silicon atom with a series of structure evolutions. In the undeformed state, silicon atom is of four-coordinate nearest neighbours. With small deformation, silicon atom maintains four coordinate neighbours having a flattening structure. As the silicon atoms experience the displacive phase transition, five-coordinate and six-coordinate crystal structures appear. Figures $3(a)$ and $(b)$ display the initial and deformed four-coordinate diamond atomic structure. 

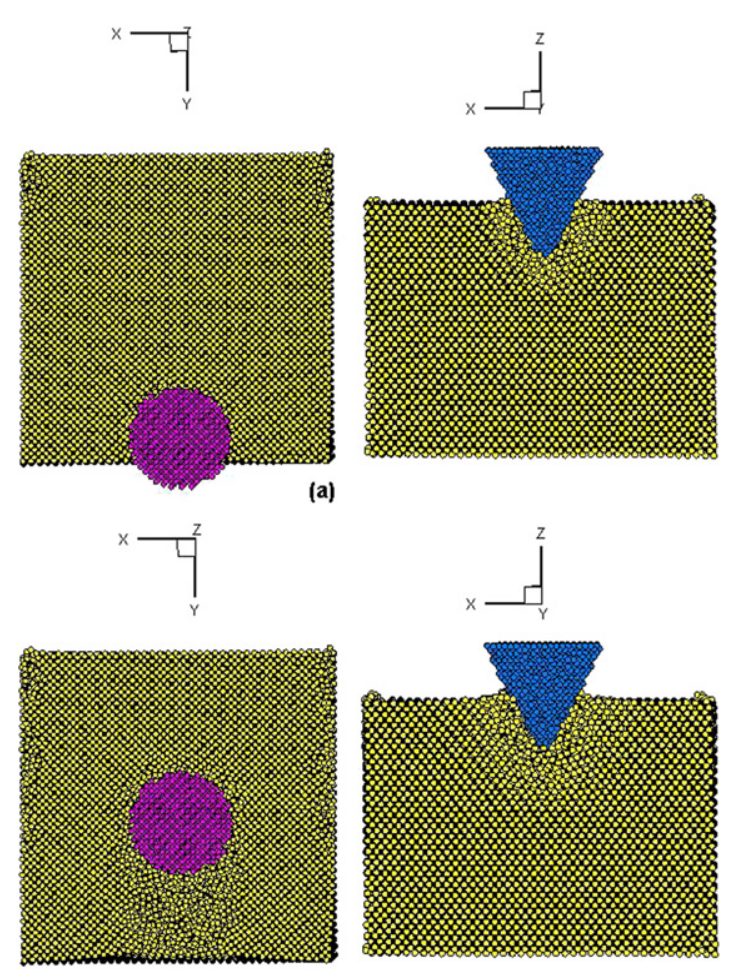

(b)
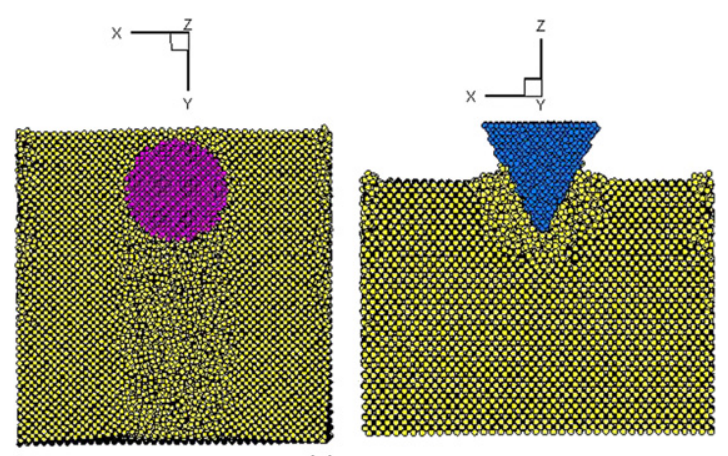

(c)
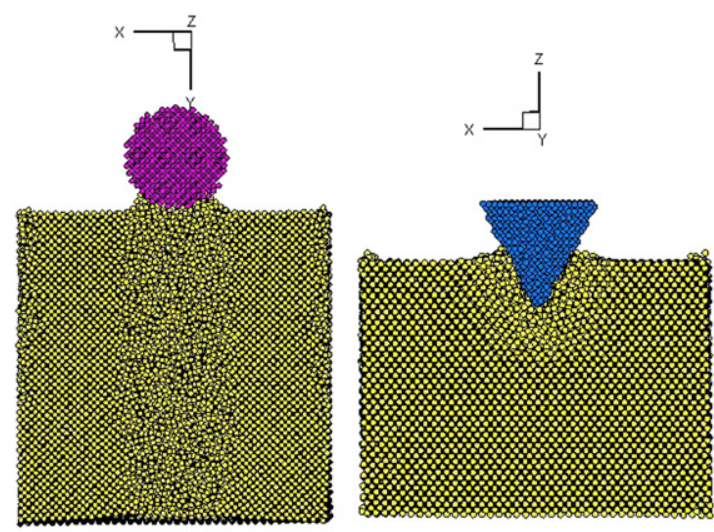

(d)

Figure 2. MD analysis at each interaction between workpiece and pin tool. A couple of snapshots show views from above and behind (a) 20000 MD steps; (b) 40000 MD steps; (c) 60000 MD steps and (d) 80000 MD steps.

Figures 3(c) and $(d)$ show the process of phase transformation at the atomic scale. As an investigation into the nature of the phase transformation, the bond length distributions of atoms is calculated. The results of these calculations are shown in

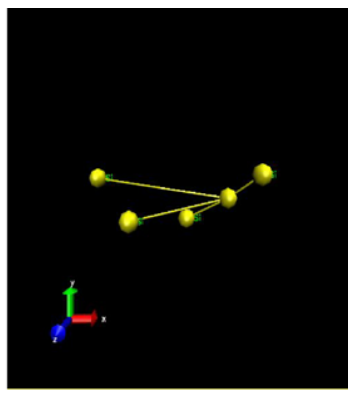

0 steps

(a)

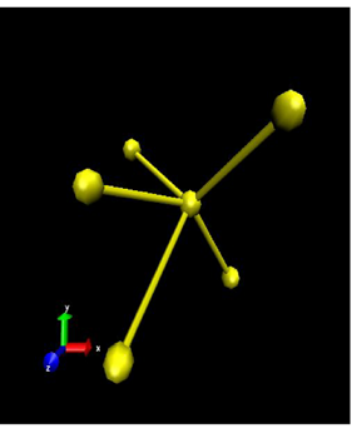

14000 steps

(c)

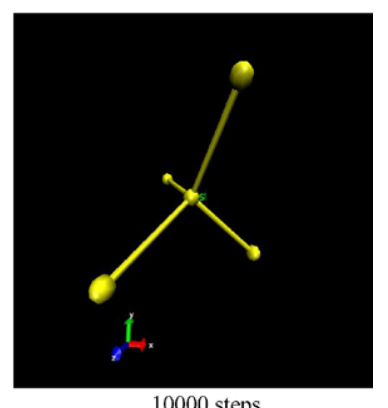

(b)

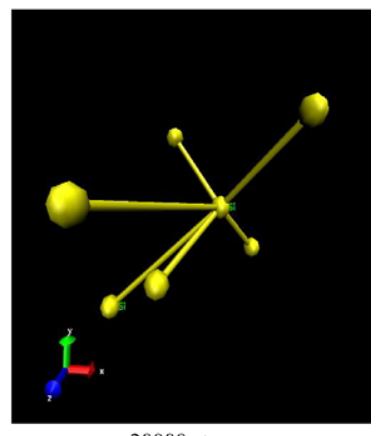

20000 steps

(d)
Figure 3. Diagram of atomic structure. (a) Tetrahedron before cutting, $(b)$ deformation tetrahedron on cutting, $(c)$ atomic structure of five coordinate during phase transition and $(d)$ deformation $\beta-$ silicon structure.

figures $4(a)$ and $(b)$. In the initial state, the bond number with a length of $2.35 \AA$ is up to maximum, and after phase transformation the bond number with a length of $2.58 \AA$ is up to local maximum. This indicates that during the cutting the average atomic distance among atoms that have undergone transformation changed from $2.35 \AA$ (diamond cubic structure) to 2.43 and $2.58 \AA$ ( $\beta$-silicon structure). Cheong and Zhang [11] performed a MD simulation with the Tersoff potential and studied phase transformation in silicon monocrystals under indentation and reported a similar result of bond number versus bond length distribution. They also calculated the maximum hydrostatic pressure $12 \mathrm{GPa}$ attained during phase transformation. The MD simulation result is supported by some experimental studies [20-22]; phase transformation in silicon from its normal, diamond cubic structure to $\beta-$ structure will take place under pressure in the range 11-13 GPa.

As a further study of the nature of phase transformation in silicon during cutting, a phase diagram analysis should be given. Both the virial pressure and temperature within the phase transformation zone under the pin tool are calculated, and the zone is constructed around the pin tool and the distance from the tool is within $1.2 \mathrm{~nm}$.

Figure 5 shows the pressure distributions versus MD steps within the phase transition zone. The curve is not smooth and the value of pressure varies significantly. Two reasons are responsible for this result. First, the phase transformation zone under pin tool is changed continuously due to the pin tool moving forward with high velocity; second, the phase transformation causes a great change in both the atomic structure and pressure. As it is about 18000 MD steps, the local average pressure may reach $12.4 \mathrm{GPa}$, which is in good 

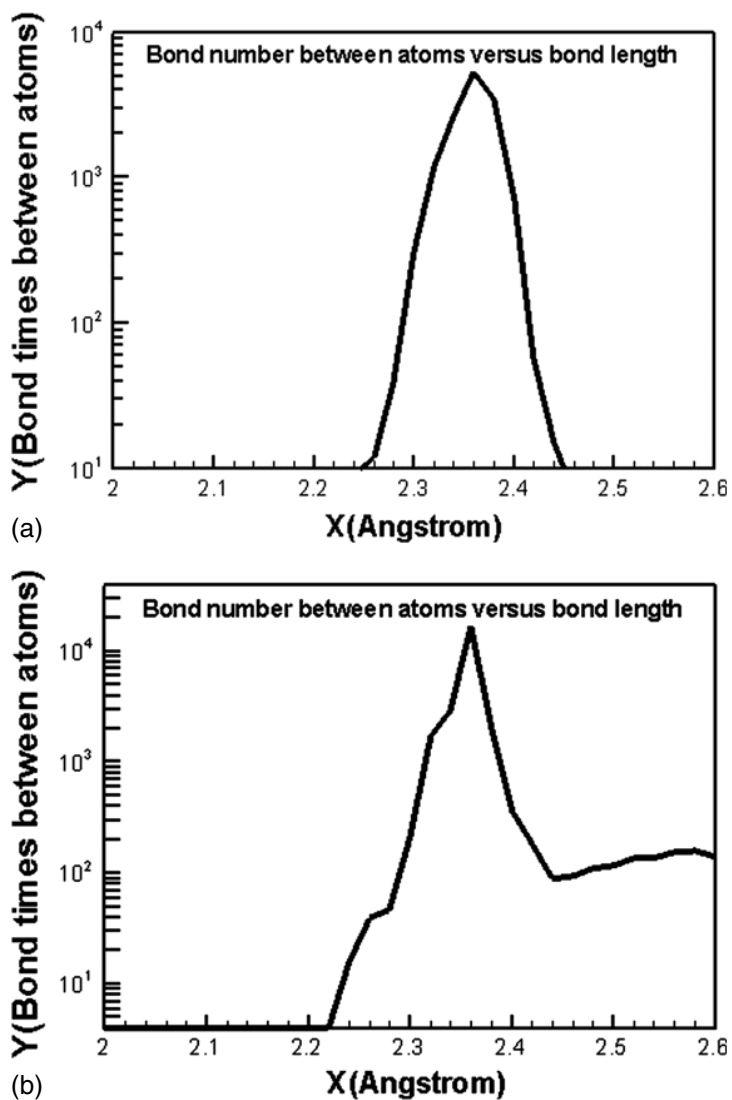

(b)

Figure 4. The number of bond versus the length of bond. (a) Start of cutting and $(b)$ the end of cutting.

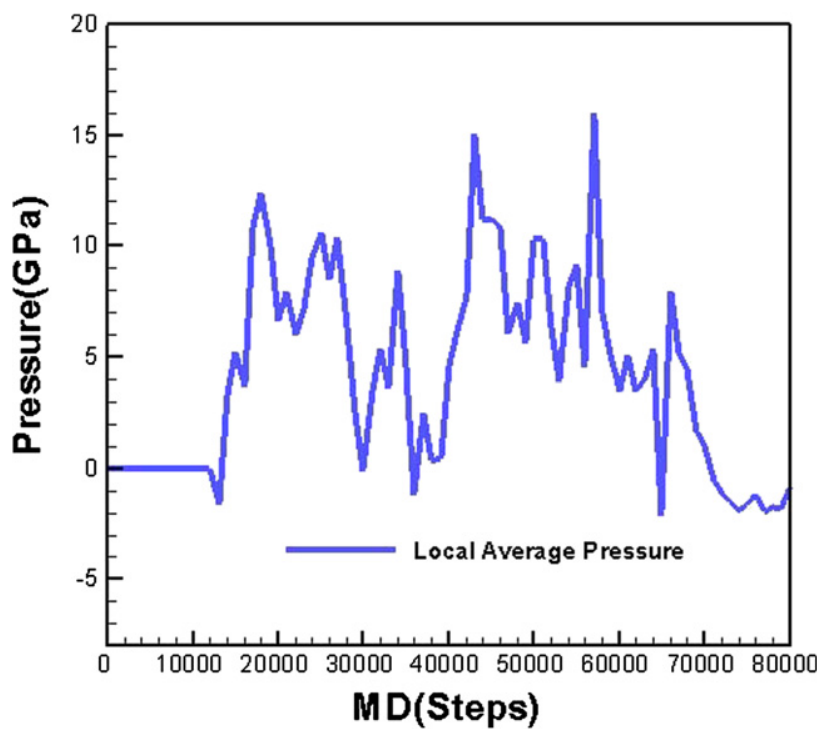

Figure 5. Pressure distribution versus MD steps.

agreement with that of [11]. The pressure $P$ is calculated according to equation (3) without numerical artefacts [23]. The curve of pressure can be a reproducible result for a given initial condition and scanning speed:

$$
P=2 N_{l}\left\langle E_{k}\right\rangle / 3 V_{l}+\left\langle\sum_{i<j} F_{i j} r_{i j}\right\rangle / 3 V_{l},
$$

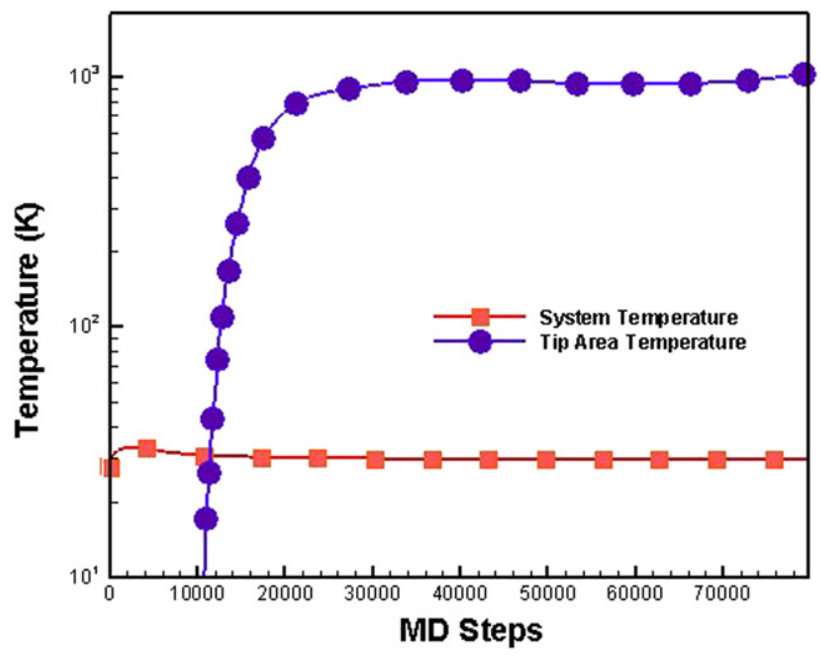

Figure 6. Comparison of temperature at the phase transformation zone under the pin tool with that of the workpiece.

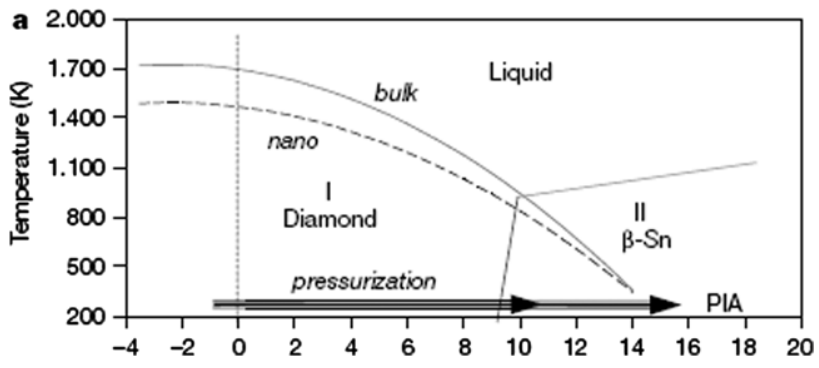

Figure 7. Temperature-pressure diagram of $\mathrm{Si}$ in stable and metastable crystalline and amorphous states.

where $N_{l}$ and $V_{l}$ are the atom number and volume of local zone, $E_{k}$ is the kinetic energy per atom, $F_{i j}$ and $r_{i j}$ are the interaction force and distance between atoms $i$ and $j$, and \langle\rangle denotes statistical averaging over all simulation time. Based on equation (4), the local temperature can be calculated [24, 25], the curve of local temperature is drawn in figure 6 , and it is about $600 \mathrm{~K}$ at $18000 \mathrm{MD}$ steps:

$$
T=\left\langle\sum_{i=1}^{N} m_{i} v_{i}^{2}\right\rangle / 3 N \kappa_{\mathrm{B}},
$$

where $\kappa_{\mathrm{B}}$ is the Boltzmann constant and $N$ is the atom number. $m_{i}$ and $v_{i}$ are the $i$ th atom mass and velocity, respectively.

Deb and his colleagues [26] studied silicon transition under high pressure with Raman spectroscopy measurements and energy-dispersive x-ray diffraction. Based on the experimental result, the temperature-pressure diagram of silicon in stable and metastable crystalline and amorphous states is calculated, and the phase diagram is shown in figure 7 [26]. Combining figures 5-7 we conclude that the phase transition occurs with a structure change from four-coordinate nearest neighbours in the diamond cubic structural to one with six-coordinate nearest neighbours in the $\beta$-silicon structure.

The relation of the number of atoms with the different coordinates nearest neighbours versus time is shown in figure 8 . At initial $4000 \mathrm{MD}$ steps relaxation, no atomic phase transition occurs. On cutting, a few silicon atoms are changed from its diamond structure to a $\beta$-silicon structure gradually. More 


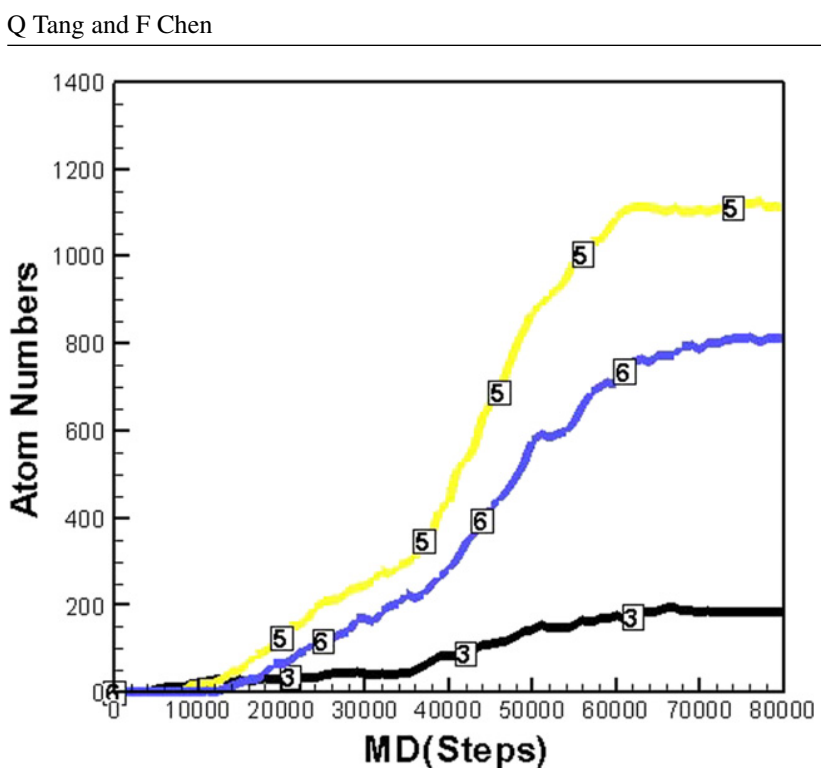

Figure 8. Number of atoms with specified nearest number of neighbours against time.

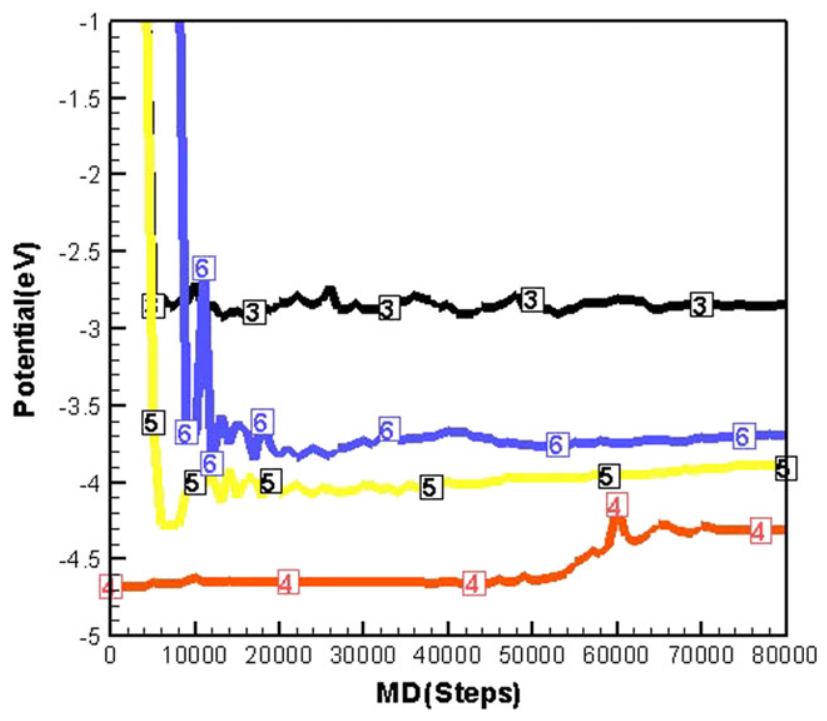

Figure 9. Potential energy evolution versus time.

silicon atoms display phase transition as the tool is in progress. The number of atoms with five-coordinate nearest neighbours is the most, and the number of atoms with three-coordinate nearest neighbours is the least. During the cutting process, the sample is in the state of compress. Some silicon atoms with three-coordinate neighbours are due to the surface effect. This implies that in the displacive phase transition process, some silicon atoms are in the flattening tetrahedron of the diamond structure, some silicon atoms complete the phase transition and are in the $\beta$-silicon structure and more atoms are in the transition state.

The potential energy variation is shown in figure 9. At the initial $6000 \mathrm{MD}$ steps, both workpiece and pin tool are in the separation state, as shown in figure 1; the potential energy is zero. From 6000 to 18000 MD steps, the potential energy varies very much, and during this period of time, the cutting process is just started, especially for the number of atoms with three-coordinate, five-coordinate and six-coordinate nearest coordinates. After 18000 steps, the potential energy curves are relatively smooth, indicative of a stable state of cutting process. After about 60000 steps, the potential energy for four-coordinate nearest neighbours is increased because the pin tool is ready to leave the workpiece.

The number of atoms with four-coordinate neighbours is subjected to small deformation; its mean potential energy per atom is about $-4.65 \mathrm{eV}$, lower than that of other potential energy curves. The mean potential energy of number of atoms with three-coordinate neighbours is of the maximum energy, up to $-2.8 \mathrm{eV}$. The potential energy of $\beta-$ silicon structure is about $-3.7 \mathrm{eV}$, far bigger than that of its original diamond structure. This result implies that a silicon atom in the $\beta$ structure state is unstable than that in the diamond structure. And one can deduce that it is not easy for a silicon atom of diamond structure undergo a phase transition to a $\beta$-silicon except under conditions of high pressure or high temperature.

\section{Conclusion}

MD simulations are carried out in order to understand the atomic scale mechanism of pin tool cutting on a semiconductor silicon. Some interesting conclusions are drawn as follows.

(1) By applying both the Tersoff and the Morse potentials, the MD simulation of a diamond pin tool cutting on the silicon workpiece is carried out. The displacive phase transition is observed. As the local temperature is up to $600 \mathrm{~K}$, the high local temperature and pressure can induce the occurrence of phase transition, and the values of pressure and temperature match the analysis of phase diagram. The simulation results of this work provide future experiments with a valuable reference, the local phase transition occurs under the pin tool during cutting.

(2) During phase transition, the form of diamond silicon is changed into a $\beta$-silicon form with high potential energy. And the local temperature during the process of phase transition is higher than the original one.

(3) No dislocation is generated for simple crystal silicon during cutting. This is due to the presence of phase transition which prevents from dislocation generation.

From this work, one can learn that when the tip is scanned with a constant load, local phase transition may occurs. It implies that the evolution of local structure at the atomic scale may lead to a change in the material behaviour. It can provide valuable reference for future application.

\section{Acknowledgments}

The research presented here was supported by the National Natural Science Foundation of China (Grant No 10372107) and Chinese Academy of Sciences (Grant No KJCX2-SWL2), and Institute of Computational Mathematics, Chinese Academy of Sciences (CAS).

\section{References}

[1] Van Loenen E J, Dijkkamp D, Hoeven A J, Lenssinck J M and Dieleman J 1989 Direct writing in Si with a scanning tunnelling microscope Appl. Phys. Lett. 55 1312-214 
[2] Mamin H J and Rugar D 1992 Thermomechanical writing with an atomic force microscope Appl. Phys. Lett. 61 1003-5

[3] Samitsu Y 1995 A study of atomic scale processing using an atomic force microscope J. Japan Soc. Precision Eng. 61 1121-5

[4] Landman U, Luedtke W D, Burnham N A and Colton R J 1990 Atomistic mechanisms and dynamics of adhesion, nanoindentation and fracture Science $\mathbf{2 4 8} 454$

[5] Maekawa K and Itoh A 1995 Friction and tool wear in nano-scale machining a molecular dynamics approach Wear 188155

[6] Mann A B, Van Heerden D and Pethica J B 2002 Contact resistance and phase transformations during nanoindentation of silicon Phil. Mag. A 821921

[7] Lorenz D, Zeckzer A, Hilpert U, Grau P, Johansen H and Leipner H S 2003 Pop-in effect as homogeneous nucleation of dislocations during nanoindentation Phys. Rev. B 67172101

[8] Kailer A, Gogotsi Y G and Nickel K G 1997 Phase transformations of silicon caused by contact loading J. Appl. Phys $\mathbf{8 1} 3057$

[9] Harrison J A, Brenner D W, White C T and Colton R J 1992 Nanoscale investigation of indention, adhesion and fracture of diamond (111) surfaces Surf. Sci. 27157

[10] Shimizu J, Eda H, Yoritsune M and Ohmura E 1998 Molecular dynamics simulation of friction on the atomic scale Nanotechnology 9118

[11] Cheong W C D and Zhang L C 2000 Molecular dynamics simulation of phase transformations in silicon monocrystals due to nano-indentation Nanotechnology 11 173-80

[12] Fang T H, Weng C T and Chang J G 2002 Molecular dynamic simulation of nano-lithography process using atomic force microscopy Surf. Sci. 501138

[13] Haile J M 1992 Molecular Dynamics Simulation: Elementary Methods (New York: Wiley)
[14] Tersoff J 1989 Modelling solid-state chemistry: Interatomic potentials for multicomponent system Phys. Rev. B 395566

[15] Tersoff J 1986 New empirical model for the structural properties of silicon Phys. Rev. Lett. 56632

[16] Stillinger F H and Weber T A 1985 Computer simulation of local order in condensed phases of silicon Phys. Rev. B 31 5262-71

[17] Allen M P and Tildesley D J 2000 Computer Simulation of Liquids (New York: Oxford University Press) p 340

[18] Lawn B R, Evans A G and Marshall D B 1980 Elastic/Plastic indentation damage in ceramics: the median/radial crack system J. Am. Ceram Soc. 63574

[19] Francois C 2000 Materials Handbook, A Concise Desktop Reference (Berlin: Springer) p 348

[20] Jang J, Lance M J, Wen S, Tsui T and Pharr G M 2005 Indentation-induced phase transformations in silicon: influences of load, rate and indenter angle on the transformation behavior Acta Mater. $\mathbf{5 3} 1759$

[21] Gerk A P and Tabor D 1978 Indentation hardness and semiconductor-metal transition of germanium and silicon Nature 271732

[22] Olijnyk H1992 Raman scattering in metallic Si and Ge up to $50 \mathrm{GPa}$ Phys. Rev. Lett. 682232

[23] Haile J M 1997 Molecular Dynamics Simulation, Element method (New York: Wiley-Interscience) p 332-9

[24] Tang Q 2004 A molecular dynamics simulation: the effect of finite size on the thermal conductivity in a single crystal silicon Mol. Phys. 102 1959-64

[25] Wang X, Hu H and Xu X 2001 Photo-acoustic measurement of thermal conductivity of thin films and bulk materials $J$. Heat Transfer 123 138-44

[26] Deb S K, Wilding M, Somayazulu M and McMillan P F 2001 Pressure-induced amorphization and an amophous-amorphous transition in densified porous silicon Nature 414528 